\title{
A review on the cross-lingual information retrieval
}

\begin{abstract}
Information retrieval involves finding some required information in a collection of information or in database. The collection not necessarily be in one language only as information does not limited to language. The simplest way to search for the information is to look at every item in the collection and when the need to translate the languages being used arises, this is where the techniques and methods that were being developed for the crosslingual retrieval system will take place. This article reviews some recent researches focusing on topics in cross-lingual information retrieval and their role in current research directions in the wide area of information retrieval.
\end{abstract}

Keyword: Cross-lingual information retrieval; Dictionary-based translation; Ontology; Query expansion 